\title{
THE STRUCTURE OF STABLE MINIMAL HYPERSURFACES IN $\mathbb{R}^{n+1}$
}

\author{
Huai-Dong Cao, Ying Shen, and Shunhui Zhu
}

\begin{abstract}
We provide a new topological obstruction for complete stable minimal hypersurfaces in $\mathbb{R}^{n+1}$. For $n \geq 3$, we prove that a complete orientable stable minimal hypersurface in $\mathbb{R}^{n+1}$ cannot have more than one end by showing the existence of a bounded harmonic function based on the Sobolev inequality for minimal submanifolds [MS] and by applying the Liouville theorem for harmonic functions due to Schoen-Yau [SY].
\end{abstract}

\section{Introduction}

This paper is concerned with the structure of complete stable minimal hypersurfaces in $(n+1)$-dimensional Euclidean space $\mathbb{R}^{n+1}$. A complete oriented minimal submanifold $M$ in $\mathbb{R}^{n+1}$ is called stable if the second variation of the volume is non-negative on any compact subset of $M$. The fundamental result along these lines is the Bernstein Theorem [B] which says that a complete areaminimizing graph in $\mathbb{R}^{3}$ is a plane. Much work has been devoted to trying to generalize it in the last thirty years. From the works of Fleming $[\mathrm{F}]$, De Giorgi [DG], Almgren [A] and J. Simons [SJ], one knows that the Bernstein Theorem is valid for complete area-minimizing graphs in $\mathbb{R}^{n+1}$ for $n \leq 7$. Counterexamples to the theorem for $n \geq 8$ were found by Bombieri-De Giorgi-Giusti [BDG] and later by Lawson $[\mathrm{L}]$. Since then, there have been attemps to extend the above Bernstein Theorem by assuming that the minimal hypersurface be stable. The best result in this direction is due to Fischer-Colbrie-Schoen [FS] and do CarmoPeng [DP], who proved that if $M^{2}$ is a complete, oriented and immersed stable minimal surface in $\mathbb{R}^{3}$, then $M$ is a plane.

However, not much is known for the geometric structure of stable minimal hypersurfaces in $\mathbb{R}^{n+1}$ when $n \geq 3$. The only known topological obstruction for stable minimal submanifolds we are aware of was a result of Schoen-Yau [SY], which states that if $M^{n}$ is a complete stable hypersurface in a manifold of non-negative curvature and $D$ is a compact domain in $M$ with smooth simply connected boundary, then there is no non-trivial homomorphism from $\pi_{1}(D)$ into the fundamental group of a compact manifold with non-positive curvature. We remark that Schoen-Yau [SY] proved the same result when $M$ is a complete

Received May 22, 1997.

1991 Mathematics Subject Classification. Primary 53C21, 53C42.

Key words and phrases. Minimal surfaces, harmonic functions, Sobolev inequality.

The first author is supported in part by NSF grant \#DMS-9504925.

The third author is supported in part by NSF grant \#DMS-9404263. 
manifold with non-negative Ricci curvature. In this paper we provide a new topological obstruction for complete stable minimal hypersurfaces in $\mathbb{R}^{n+1}$. Our main result can be stated as follows:

Theorem 1. For any $n \geq 3$, if $M^{n}$ is a complete non-compact oriented stable minimal hypersurface in $\mathbb{R}^{n+1}$, then $M$ has only one end.

To our knowledge, this result is new even for area-minimizing hypersurfaces $M^{n}$ in $\mathbb{R}^{n+1}$ for $n \geq 8$. Note that the stable condition in Theorem 1 cannot be dropped, since a catenoid, which is unstable, clearly has two ends. It is also clear that our result differs from Schoen-Yau's theorem. For example, our result says that any manifold of topological type $N^{n-1} \times \mathbb{R}$ with $N^{n-1}$ compact cannot be a stable minimal hypersurface in $\mathbb{R}^{n+1}$. In fact, Theorem 1 can be compared with the similar result of Gromoll-Meyer [GM] for complete manifolds with positive Ricci curvature.

The proof of our main theorem relies on the Sobolev ineqality for minimal submanifolds due to Michael and Simon [MS] and the Liouville theorem for harmonic maps due to Schoen and Yau [SY]. One crucial step in the proof is to show the existence of a non-trivial bounded harmonic function with finite Dirichlet energy in case the minimal hypersurface has more than one end. This is done by using the Sobolev inequality together with a choice of cut-off functions based on the fact that the minimal submanifold has more than one end. We remark that our cut-off function actually has noncompact support. This non-standard choice of cut-off functions allows us to aviod assumptions such as volume doubling properties and volume growth conditions of the ends.

Finally, we would like to point out that the method we used in the proof of Lemma 2 in next section yields the following result which is of independent interest:

Theorem 2. Let $M^{n}$ be a complete noncompact Riemannian manifold with at least two ends of infinite volume. Suppose that either

(1) the Sobolev inequality holds on $M$, or

(2) the first eigenvalue $\lambda_{1}(M)$ of $M$ is positive.

Then there exists on $M$ a non-constant bounded harmonic function with finite Dirichlet energy.

The problem of the existence of harmonic functions on a complete manifold has a long history. For some of the recent progress on this problem, we refer the readers to the important works of Li-Tam ([LT1] and [LT2]), Colding-Minicozzi ([CM1], [CM2], [CM3] and [CM4]) and a very recent survey article of Peter Li $[\mathrm{Li}]$.

\section{The Proof}

In this section we prove Theorem 1 stated in the introduction. First let us fix some notations and recall the definition of a stable minimal submanifold. 
Let $\left\{U_{i}\right\}_{i=1}^{\infty}$ be a family of relatively compact open sets which exhaust the manifold $M$, i.e.,

$$
\begin{gathered}
U_{i} \subset U_{i+1}, \\
\cup_{i=1}^{\infty} U_{i}=M .
\end{gathered}
$$

An end of $M$ is an inverse system $E=\left\{E^{(i)}\right\}_{i=1}^{\infty}$ such that

$$
E^{(i+1)} \subset E^{(i)}
$$

and $E^{(i)}$ is a connected component of $M \backslash \bar{U}_{i}$.

Remark 2. If $M$ has only finitely many ends, then there is some $i_{0}>0$ such that all inverse systems $\left(E^{(i)}\right)$ stabilize for $i \geq i_{0}$, i.e., $E^{(i)}=E^{\left(i_{0}\right)}$.

Remark 3. An end can also be defined as equivalent classes of cofinal curves, where two curves $c_{1}, c_{2}:[0, \infty) \rightarrow M$ are cofinal if for every compact set $K \subset$ $M$ there is some $t>0$ such that $c_{1}\left(t_{1}\right)$ and $c_{2}\left(t_{2}\right)$ lie in the same connected component of $M \backslash K$ for all $t_{1}, t_{2}>t$.

Let $M^{n}$ be a complete oriented submanifold minimally immersed in $\mathbb{R}^{n+1}$. $M$ is said to be stable if the second variation of the volume is non-negative on any compact subset of $M$. More precisely, let $e_{1}, e_{2}, \cdots, e_{n+1}$ be a positively oriented orthonormal frame on $M$ with $e_{1}, \cdots, e_{n}$ tangential, and $e_{n+1}$ the globally defined unit normal vector to $M$. The second fundamental form $\left\{h_{i j}\right\}$ of $M$ is defined by

$$
h_{i j}=\left\langle\nabla_{e_{i}} e_{n+1}, e_{j}\right\rangle
$$

for $i, j=1,2, \cdots, n$, where $\nabla$ is the Riemannian connection of $\mathbb{R}^{n+1}$. $M$ is minimal if the mean curvature

$$
H=\sum_{i=1}^{n} h_{i i}=0 .
$$

The stability condition of $M$ is given by the following inequality (see [SJ] or $[\mathrm{SY}])$ :

$$
\int_{M}|\nabla \phi|^{2} \geq \int_{M} \sum_{i, j=1}^{n} h_{i j}^{2} \phi^{2}
$$

where $\phi$ is any function with compact support on $M$.

Now we are ready to present the proof of Theorem 1 .

Lemma 1. If $M^{n}$ is a complete orientable minimal hypersurface in $\mathbb{R}^{n+1}$, then every end of $M^{n}$ has infinite volume.

Proof: In fact we will show that for any compact set $K \subset M$, every noncompact component of $M \backslash K$ has infinite volume. Let $E$ be a component of $M \backslash K$. We will adopt an argument of Yau $([\mathrm{Y}])$ to the end $E$. 
Take an arbitrary point $p \in M$, without loss of generality, we may assume $p=0$. In the following we let $d(\cdot, \cdot)$ be the distance function of $\mathbb{R}^{n+1}$, and $r(\cdot, \cdot)$ the distance function of $M$ with respect to the induced metric. We will write $d(x), r(x)$ if the base point is 0 . Obviously $d \leq r$ for any two points in $M$. Let $\gamma$ be a minimal geodesic from 0 , then,

$$
\begin{aligned}
\frac{\partial d}{\partial r} & =\lim _{t \rightarrow 0} \frac{d(\gamma(s+t))-d(\gamma(s))}{t} \\
& \leq \lim _{t \rightarrow 0} \frac{d(\gamma(s+t), \gamma(s))}{t} \quad \text { ( by the triangle inequality) } \\
& \leq 1 . \quad \text { (since } d \leq r)
\end{aligned}
$$

By a direct computation and using the fact that $M$ is minimal, one can show that

$$
\triangle_{M} d^{2}(x)=2 n \text {. }
$$

Let $B(s)$ be the geodeisc ball of $M$, of radius $s$ centered at 0 . Integrating the above equation over $B(s)$ and using (1), we obtain

$$
2 n \operatorname{vol}(B(s)) \leq 2 s \operatorname{vol}(\partial B(s)) .
$$

Note that in any manifold,

$$
\operatorname{vol}(\partial B(s))=\left.\frac{\partial}{\partial r}\right|_{r=s} \operatorname{vol}(B(s)) .
$$

We thus obtain

$$
\left.s \frac{\partial}{\partial r}\right|_{r=s} \operatorname{vol}(B(r))-n \operatorname{vol}(B(s)) \geq 0,
$$

which implies $s^{-n} \operatorname{vol}(B(s))$ is non-decreasing. Therefore

$$
\frac{\operatorname{vol}(B(s))}{s^{n}} \geq \lim _{s \rightarrow 0} \frac{\operatorname{vol}(B(s))}{s^{n}}=\omega(n)
$$

where $\omega(n)$ is the volume of unit ball in $\mathbb{R}^{n}$.

Now if $E$ has finite volume, choose $R$ big enough such that

$$
\omega(n) R^{n}>\operatorname{vol}(E) .
$$

Let $p$ be a point in $E$ such that $r(p, \partial E) \geq R$, then

$$
\operatorname{vol}(E) \geq \operatorname{vol}(B(R)) \geq \omega(n) R^{n}>\operatorname{vol}(E),
$$

a contradiction.

Lemma 2. Let $M^{n}$ be a complete orientable minimal hypersurface in $\mathbb{R}^{n+1}$ with at least two ends. Then there exists on $M$ a non-constant bounded harmonic function with finite energy.

We remark that the statement of Lemma 2 still holds when $M^{n}$ is a complete submanifold in $\mathbb{R}^{n+m}$ for $n \geq 3, m \geq 1$ provided one of the following conditions holds: (1) $\int_{M}|\vec{H}|^{n}<1 / c_{n}^{\frac{n}{2}}$; or (2) $\int_{M}|\vec{H}|^{\frac{4 n}{n+2}}<\infty$. Here $\vec{H}$ is the mean 
curvature vector of $M$ and $c_{n}$ is a (Sobolev) constant which depends only on the dimension $n$.

Proof of Lemma 2: In the following, we will use an exhaustion of $M$ by compact submanifolds with boundary. The obvious choice is the distance balls which are not smooth in general. It is standard to smooth the distance function to a point $p$ by, say, local averaging, to get a smooth function $f$. $f$ still has compact sublevel sets. By Sard's Theorem, we can choose a sequence of regular values $\left\{R_{i}\right\}$ of $f$, such that $\left\{f^{-1}\left(0, R_{i}\right)\right\}$ gives an exhausion of $M$ by compact smooth submanifolds. In the following, we denote $D_{i}=f^{-1}\left(0, R_{i}\right)$. We shall also use the notations for the ends of $M$ introduced at the begining of this section. For $i \geq i_{0}$ and $i_{0}$ sufficiently large, let

$$
M \backslash D_{i}=\cup_{j=1}^{s} E_{j}^{(i)}
$$

be the disjoint union of connected components, with $s \geq 2$.

By Lemma 1 and the assumptions in Lemma 2, $M$ has at least two components with infinite volume. Let $E_{1}^{\left(i_{0}\right)}, E_{2}^{\left(i_{0}\right)}$ be two such components. On each compact domain $D_{i}$, we can minimize the energy functional $\int_{D_{i}}|\nabla u|^{2} d x$ among all functions $u$ such that $\left.u\right|_{\partial E_{1}^{(i)}}=1$ and $\left.u\right|_{\partial E_{j}^{(i)}}=0$ for all $j \geq 2$, where $\nabla$ and $d x$ are gradient and volume element of $M$, respectively. We denote the minimizer by $u_{i}$. Then $u_{i}$ is the unique solution of the following Dirichlet problem on $D_{i}$

$$
\left\{\begin{array}{l}
\triangle u(x)=0 \\
\left.u\right|_{\partial E_{1}^{(i)}}=1 \\
\left.u\right|_{\partial E_{j}^{(i)}}=0 .(j \neq 1)
\end{array}\right.
$$

By the maximum principle, we have $0 \leq u_{i} \leq 1$ on $D_{i}$. Moreover, it is easy to see that $\int_{D_{i}}\left|\nabla u_{i}\right|^{2} d x \leq \int_{D_{j}}\left|\nabla u_{j}\right|^{2} d x$ for $i>j$ and hence there is a universal constant $C_{1}>0$ such that

$$
\int_{D_{i}}\left|\nabla u_{i}\right|^{2} d x<C_{1}
$$

Therefore by passing to a subsequence, still denoted by $u_{i}$, we can find a harmonic function $u$ on $M$ such that

$$
\lim _{i \rightarrow \infty} u_{i}(x)=u(x), \quad x \in M
$$

and

$$
\int_{M}|\nabla u|^{2} d x<C_{1}
$$

It is clear from the construction that $0 \leq u(x) \leq 1$ on $M$.

In the following we prove that the limiting harmonic function $u$ is not a constant function. We will prove this by contradiction. 
By using the Sobolev inequality for minimal hyperfaces in $\mathbb{R}^{n+1}$ (see [MS]), we have, for any smooth function $\phi$ which vanishes on $\partial D_{i}$,

$$
\left(\int_{D_{i}} \phi^{p}(x) d x\right)^{\frac{2}{p}} \leq c_{n} \int_{D_{i}}|\nabla \phi|^{2} d x
$$

where $p=2 n / n-2$ (this is where we need to assume $n \geq 3$ ) and $c_{n}$ is the Sobolev constant which only depends on the dimension $n$.

Note that from the construction of $u_{i}$, the function $u_{i}\left(1-u_{i}\right)$ vanishes on $\partial D_{i}$. Setting $\phi=u_{i}\left(1-u_{i}\right)$ in $(4)$, we obtain

$$
\begin{aligned}
\left(\int_{D_{i}}\left(u_{i}\left(1-u_{i}\right)\right)^{p} d x\right)^{\frac{2}{p}} & \leq c_{n} \int_{D_{i}}\left|\nabla u_{i}-2 u_{i} \nabla u_{i}\right|^{2} d x \\
& \leq c_{n} \int_{D_{i}}\left(\left|\nabla u_{i}\right|+2 u_{i}\left|\nabla u_{i}\right|\right)^{2} d x \\
& \leq 9 c_{n} \int_{D_{i}}\left|\nabla u_{i}\right|^{2} d x \leq 9 c_{n} C_{1} .
\end{aligned}
$$

Since $\operatorname{vol}\left(D_{i}\right) \rightarrow \infty$, by letting $i \rightarrow \infty$ in (5), it follows that if $u$ is a constant function, then $u \equiv 0$ or $u \equiv 1$.

Thus we only need to show that $u$ cannot be identically 0 or 1 . If $u \equiv 0$, we may replace $u_{i}$ and $u$ by $\hat{u}_{i}=1-u_{i}$ and $\hat{u}=1-u$, respectively. Then $\hat{u} \equiv 1$ and furthermore, $\hat{u}_{i}$ satisfies (3), and (5). Thus we may assume that $u \equiv 1$. (This is where we use the condition that the manifold has at least two ends with infinite volume.)

We choose a smooth function $\psi$ such that

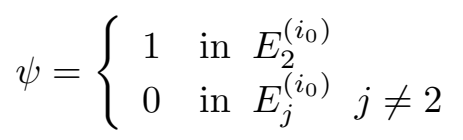

and

$$
|\nabla \psi| \leq C_{2}, \quad 0 \leq \psi \leq 1
$$

for some constant $C_{2}>0$ which is independent of $i$ and $u_{i}$. Note that $|\nabla \psi|$ vanishes outside a compact set.

Since $\left.u_{i}\right|_{E_{1}^{(i)}}=1$ and $\left.u_{i}\right|_{E_{j}^{(i)}}=0$ for $j \geq 2$, the function $\phi=u_{i} \psi$ vanishes on $\partial D_{i}$. The Sobolev inequality (5) implies that

$$
\begin{aligned}
\left(\int_{D_{i}}\left(u_{i} \psi\right)^{p} d x\right)^{\frac{2}{p}} & \leq c_{n} \int_{D_{i}}\left|\nabla\left(u_{i} \psi\right)\right|^{2} d x \\
& \left.\leq c_{n} \int_{D_{i}}\left|\psi \nabla u_{i}\right|+u_{i}|\nabla \psi|\right)^{2} d x \\
& \leq 2 c_{n}\left(\int_{D_{i}} \psi^{2}\left|\nabla u_{i}\right|^{2}+\int_{D_{i}}|\nabla \psi|^{2}\right) \leq C_{3}
\end{aligned}
$$

where $C_{3}$ is a constant independent of $i$ and $u_{i}$. 
Therefore, we have

$$
\int_{E_{2}^{\left(i_{0}\right)} \cap D_{i}}\left(u_{i}\right)^{p} \leq C_{3}
$$

for all $i \geq i_{0}$. Letting $i \rightarrow \infty$, we get

$$
\operatorname{vol}\left(E_{2}^{\left(i_{0}\right)}\right)=\int_{E_{2}^{\left(i_{0}\right)}} u^{p} \leq C_{3}
$$

This contradicts our assumption that $\operatorname{vol}\left(E_{2}^{\left(i_{0}\right)}\right)$ is infinite. Therefore the limiting harmonic function $u$ is not a constant function.

Lemma 3. (Schoen-Yau [SY]) Let $M$ be a complete noncompact stable minimal hypersurface in a manifold of non-negative curvature. If $u$ is a harmonic function on $M$ with bounded energy, then $u$ is constant.

This is a special case of the Liouville Theorem for harmonic map that Schoen and Yau originally proved.

Now suppose $M^{n}(n \geq 3)$ is a complete, oriented stable minimal hypersurface in $\mathbb{R}^{n+1}$ with finitely many ends. From Lemma 1 we know that each end of $M$ has infinite volume. If $M$ has more than one end then Lemma 2 implies that $M$ supports a non-constant harmonic function with finite energy. This is a contradiction to Lemma 3 . Hence $M$ has only one end and the proof of Theorem 1 is completed.

\section{Acknowledgements}

We would like to thank Prof. Richard Schoen for bringing this problem to our attention and for very helpful discussions. We would also like to thank Professors Jeff Cheeger, Peter Li, and S.-T. Yau for their interest in this work.

\section{References}

[A] F. J. Almgren, Jr., Some interior regularity theorems for minimal surfaces and an extension of Bernstein's theorem, Ann. of Math., 84 (1966), 277-292.

[B] S. Bernstein, Sur un théorème de Gémétrie et ses applications aux équations aux dérivées partielles du type elliptique, Comm. de la Soc. Math. de Kharkov (2é sér.) 15 (1915-1917), 38-45.

[BDG] E. Bombieri, E. De Giorgi, and E. Giusti, Minimal cones and the Bernstein problem, Invent. Math. 7 (1969), 243-268.

[Ch-E] J. Cheeger and D. G. Ebin, Comparison Theorems in Riemannian Geometry, NorthHolland Mathematical Library, Vol. 9., North-Holland Publishing Company, 1975.

[CM1] T. Colding and W. Minicozzi, Generalized Liouville properties for manifolds, preprint

[CM2] — Harmonic functions on manifolds, preprint

[CM3] _ Weyl type bounds for harmonic functions, preprint

[CM4] _ Liouville theorems for harmonic sections and applications manifolds, preprint

[DG] E. De Giorgi, Una estensione del teorema di Berstein, Ann. Scuola Norm. Sup. Pisa 19 (1965), 79-85. 
[DP] M. do Carmo and C. K. Peng, Stable complete minimal surfaces in $R^{3}$ are planes, Bull. Amer. Math. Soc., 1 (1979), 903-906.

[F] W. Fleming, On the oriented Plateau problem, Rend. Circ. Mat. Palerino 11 (1962), 69-90.

[FS] D. Fischer-Colbrie and R. Schoen, The structure of complete stable minimal surfaces in 3-manifolds of non-negative scalar curvature, Comm. Pure Appl. Math. 33 (1980), $121-132$.

[GM] D. Gromoll and W. Meyer, On complete open manifolds of positive curvature, Ann. of Math. 90 (1969), 75-90.

[L] B. Lawson, The equivariant Plateau problem and interior regularity, Trans. Amer. Math. Soc. 173 (1972), 231-250.

[Li] P. Li, Curvature and function theory on Riemannian manifolds, preprint.

[LT1] P. Li and L. F. Tam, Positive harmonic functions on complete manifolds with nonnegative curvature outside a compact set, Ann. of Math. 125 (1987), 171-207

[LT2] _ Harmonic functions and the structure of complete manifolds, J. Diff. Geom. 35 (1992), 359-383.

[MS] J. Michael and L. M. Simon, Sobolev and mean-value inequalities on generalized submanifolds of $R^{n}$, Comm. Pure Appl. Math. 26 (1973), 361-379.

[SJ] J. Simons, Minimal varieties in Riemannian manifolds, Ann. of Math. 88 (1968), $62-105$.

[SY] R. Schoen and S.-T. Yau, Harmonic maps and the topology of stable hypersurfaces and manifolds with nonnegative Ricci curvature, Comment. Math. Helvetici 51 (1976), 333-341.

[Y] S.-T. Yau, Isoperimetric constants and the first eigenvalue of a compact Riemannian manifold, Ann. Sci. Ècole Norm. Sup., 8 (1975), 487-507.

Department of Mathematics, Texas A\&M University, College Station, TX 77843

E-mail address: cao@math.tamu.edu

Department of Mathematics, Dartmouth College, Hanover, NH 03755

E-mail address: Ying.Shen@dartvax.dartmouth.edu, Shunhui.Zhu@dartvax.dartmouth.edu 